\title{
No link between probiotics and obesity? Author reply
}

\section{Didier Raoult}

I am surprised that my Editorial (Probiotics and obesity: a link? Nature Rev. Microbiol. 7, $616(2009))^{1}$, in which I proposed a possible link between probiotics and obesity, would generate such animated correspondence from such well-known scientists as Ehrlich (Probiotics - little evidence for a link to obesity. Nature Rev. Microbiol. 7, 16 November 2009 (doi:10.1038/nrmicro209-c1) $)^{2}$ and Delzenne and Reid (No causal link between obesity and probiotics. Nature Rev. Microbiol. 7, 16 November 2009 (doi:10.1038/nrmicro2209-c2) $)^{3}$. Surprisingly, Ehrlich confirms that a valid hypothesis exists simply by stating that he will investigate this query in the future.

These comments on my Editorial provide a good opportunity to update my position on this question. Indeed, it is difficult to ignore previous work showing how transplantation of gut microbiota from obese mice to bacteria-free mice can cause obesity in the recipients ${ }^{4}$.

For many years, the manipulation of gut microbiota by growth promoters, including probiotics, prebiotics and antibiotics, has had a huge impact on the livestock and poultry industries, ever since they were first prescribed to prevent diarrhoea in animals and later found to be useful for weight gain ${ }^{5}$. One needs only to perform a quick search on the internet using the key words 'growth promoters' and 'probiotics' to see the enormity of this market. My interest in a possible link between Lactobacillus spp. and obesity evolved from some paradoxical results that we identified in birds following their intragastric inoculation with lactobacilli ${ }^{6}$, and I remain convinced that this link requires further investigation ${ }^{7}$. Since the publication of my Editorial ${ }^{1}$, our group has published a report showing that the prevalence of Lactobacillus spp. in the intestinal tracts of obese persons is notably greater than the prevalence observed in lean individuals ${ }^{8}$.

Certainly, more work is needed to evaluate the full effects of probiotics on humans; however, preliminary data suggest that children gain weight when given probiotics. In some of these initial studies, the small number of children enrolled precluded a statistically significant assessment. However, in one long term study, the weight gain in infants receiving probiotics was $4 \%$ compared with controls, for three different products administered to male or female infants ${ }^{9}$. This increase is similar to the weight gain that was observed in piglets receiving probiotics $^{10}$. All six studies with probiotics show a weight gain of $\geq 3 \%$ compared with controls (Fisher exact test $p=0.04)^{9}$. Furthermore, one of the references mentioned by Ehrlich that supposedly opposes the notion that probiotics promote weight gain is not related to the subject $^{11}$.

In conclusion, there is enough evidence from the use of probiotics as growth promoters in animals and from preliminary studies in humans to consider a link between probiotics and obesity. I am happy to see that this hypothesis will be tested by the European Commission project on Metagenomics of the Human Intestinal Tract (MetaHIT), as stated by Ehrlich²; I am also pursuing this type of evaluation. A final determination will undoubtedly require more work. Nonetheless, the idea that probiotics may cause effects in humans that are similar to those observed in many other species of animals cannot be that surprising. We must keep an open mind when dealing with the many causes of obesity in the twenty-first century.
Didier Raoult is at Unité de Recherche en Maladies Infectieuses et Tropicales Emergentes, CNRS-IRD 6236, Faculté de Médecine, Université de la Méditerranée, 27 Boulevard Jean Moulin, 13385 Marseille, France. e-mail: didier.raoult@gmail.com doi:10.1038/nrmicro2209-c3

1. Raoult, D. Probiotics and obesity: a link? Nature Rev. Microbiol. 7, 616 (2009).

2. Erhlich, S. D. Probiotics - little evidence for a link to obesity. Nature Rev. Microbiol. 7, 16 November 2009 (doi: 10.1038/nrmicro2209-c1).

3. Delzenne, N. \& Reid, G. No causal link between obesity and probiotics. Nature Rev. Microbiol. 7, 16 November 2009 (doi: 10.1038/nrmicro2209-c2).

4. Turnbaugh, P. J. et al. An obesity-associated gut microbiome with increased capacity for energy harvest. Nature 444, 1027-1131 (2006).

5. Anadon, A., Martinez-Larranaga, M. R. \& Aranzazu, M. M. Probiotics for animal nutrition in the European Union. Regulation and safety assessment. Regul. Toxicol. Pharmacol. 45, 91-95 (2006).

6. Khan, M., Raoult, D., Richet, H., Lepidi, H. \& La Scola, B. Growth-promoting effects of single-dose intragastrically administered probiotics on chicks. Br. Poult. Sci. 48, 732-735 (2007).

7. Raoult, D. Obesity pandemics and the modification of digestive bacterial flora. Eur. J. Clin. Microbiol. Infect. Dis. 27, 631-634 (2008).

8. Armougom, F., Henry, M., Vialettes, B., Raccah, D. \& Raoult, D. Monitoring bacterial community of human gut microbiota reveals an increase in Lactobacillus in obese patients and Methanogens in anorexic patients. PLOS ONE 4, e7125 (2009).

9. Chouraqui, J. P. et al. Assessment of the safety, tolerance, and protective effect against diarrhea of infant formulas containing mixtures of probiotics or probiotics and prebiotics in a randomized controlled trial. Am. J. Clin. Nutr. 87, 1365-1373 (2008).

10. Fajardo Bernardez, P., Fucinos Gonzalez, C., Mendez Batan, J., Pastrana Castro, L. \& Perez Guerra, N. Performance and intestinal coliform counts in weaned piglets fed a probiotic culture (Lactobacillus casei subsp. casei CECT 4043) or an antibiotic. J. Food Prot. 71, 1797-1805 (2008).

11. Le Bellego, L. \& Noblet, J. Performance and utilization of dietary energy and amino acids in piglets fed low protein diets. Livest. Prod. Sci. 76, 45-58 (2002). 